\title{
ON CIRCULARLY SYMMETRIC FUNCTIONS
}

\author{
JAMES A. JENKINS
}

1. In addition to the study devoted to the general class of normalized regular univalent functions in the unit circle considerable attention has been given to certain subclasses of these functions such as starlike functions, convex functions, and others. In view of the important role played by symmetrization methods in recent work it seems worth while to study a further subclass - the class of circularly symmetric functions. These functions map the unit circle on a domain of the type obtained by application of the process of circular symmetrization. In the present paper these functions are shown to admit a characterization of the same type as the familiar one for starlike functions. From this it follows that a circularly symmetric function maps the concentric subcircles of the unit circle onto circularly symmetric domains. Further these functions are found to be related to the class of typically real functions introduced by Rogosinski [4] in essentially the same way as starlike functions are related to the class of normalized regular functions with positive real part. From this fact can be drawn some interesting consequences concerning coefficients and function values associated with the class of circularly symmetric functions.

2. Let us denote by $S$ the class of functions $f(z)$ regular and univalent for $|z|<1$ which have power series expansion about $z=0$ beginning

$$
f(z)=z+a_{2} z^{2}+\cdots .
$$

Let $D$ be a domain in the $w$-plane containing the point $w=0$ and having the following structure. Let the intersection of $D$ with the circle $|w|=R$ consist of the whole circle for $R<R_{0}, R_{0}>0$ and of the arc $-l(R)<\Phi<l(R)$ for $R_{0} \leqq R<R_{1}$ where $0<l(R) \leqq \pi$ and $R_{1}$ may be $\infty$. If $R_{1}$ is not infinite the intersection of $D$ with $|w|=R, R \geqq R_{1}$ is to be void. Here $R, \Phi$ are polar coordinates in the $w$-plane. We include the special case that $D$ is the circle $|w|<R_{0}$. Such a domain $D$ is called a circularly symmetric domain. Domains of this type are obtained by applying the process of circular symmetrization with respect to the positive real axis to any simply-connected domain containing $w=0$. In the present work we shall always understand circular symmetrization in this sense. Let now $Y$ denote the subclass of functions of $S$

Received by the editors October 22, 1954. 
which map $|z|<1$ onto a circularly symmetric domain. Such functions will be called circularly symmetric functions. Further let $T$ denote the class of functions $f(z)$ regular for $|z|<1$, normalized by the condition (1) and such that $f(z)$ is real for real $z$ with $|z|<1$ and for these values only. These are typically real functions in the sense of Rogosinski. Since a function in $Y$ is univalent and has real coefficients in its Taylor expansion about the origin we recognize that $Y$ is a subclass of $T$.

Theorem 1. If $f \in Y$ and $|z|<1$

$$
\begin{array}{ll}
\Im\left\{z f^{\prime}(z) / f(z)\right\} \geqq 0, & \Im z \geqq 0, \\
\Im\left\{z f^{\prime}(z) / f(z)\right\} \leqq 0, & \Im z \leqq 0 .
\end{array}
$$

First let us suppose that $f(z)$ can be extended continuously to $|z|=1$ to map that circle into a smooth curve. Then by the definition of a circularly symmetric domain we have that $\left|f\left(e^{i 0}\right)\right|$ decreases as $\theta$ increases from 0 to $\pi$, that is

$$
\frac{d}{d \theta} \log \left|f\left(e^{i \theta}\right)\right| \leqq 0, \quad 0 \leqq \theta \leqq \pi .
$$

Rewriting this successively as

$$
\begin{array}{r}
\frac{d}{d \theta} \Re\left\{\log f\left(e^{i \theta}\right)\right\} \leqq 0, \\
\Re\left\{\frac{d}{d \theta} \log f\left(e^{i \theta}\right)\right\} \leqq 0,
\end{array}
$$

and observing that $d \log f\left(e^{i \theta}\right) / d \theta$ are the continuous boundary values of

$$
\frac{d \log f(z)}{d z} \cdot \frac{\partial z}{\partial \theta}=i z f^{\prime}(z) / f(z)
$$

while $\Im\left\{z f^{\prime}(z) / f(z)\right\}=0$ for $\Im z=0$, we see that we have

$$
\Im\left\{z f^{\prime}(z) / f(z)\right\} \geqq 0 \text { for } \Im_{z} \geqq 0 .
$$

The second part of (2) follows in the same way.

To extend this result so as to eliminate the boundary restriction we do not have available the elegant device of Radó [3] so we use a proof more like that given by Nevanlinna [2]. First of all we note that the function $l(R), R_{0} \leqq R<R_{1}$, associated with the image domain $D$ of $|z|<1$ under $f(z)$ is lower semi-continuous (since $D$ is open), thus we can find a sequence of functions $l_{n}(R), R_{0} \leqq R<R_{1}$, possessing 
any desired degree of differentiability and such that $l_{n}(R) \leqq l(R)$ and

$$
\lim _{n \rightarrow \infty} l_{n}(R)=l(R), \quad \quad R_{0} \leqq R<R_{1} .
$$

The special case where $D$ is the circle $|w|<1$ is included in the previous treatment, thus we may assume $R_{0}<R_{1}$. Let $R_{0}^{(n)}$ be a sequence of values, $n=1,2, \cdots, R_{0}^{(n)}<R_{1}$, decreasing to $R_{0}$ as $n$ approaches infinity. Let $R_{1}^{(n)}$ be a sequence of values, $n=1,2, \cdots, R_{0}^{(1)}<R_{1}^{(n)}$ $<R_{1}$, increasing to $R_{1}$ as $n$ approaches infinity. Let $\rho_{n}$ be numbers satisfying $0<\rho_{n}<R_{1}-R_{1}^{(n)}$, thus being understood in the obvious sense if $R_{1}$ is infinite. Let $D_{n}$ be the simply-connected domain whose intersection with the circle $|w|=R$ consists of the whole circle for $R<R_{0}$, of the arc $-\lambda_{n}(R)<\Phi<\lambda_{n}(R)$ for $R_{0} \leqq R<R_{1}^{(n)}+\rho_{n}$ and is void for $R \geqq R_{1}^{(n)}+\rho_{n}$. The function $\lambda_{n}(R)$ is to satisfy $0<\lambda_{n}(R) \leqq \pi$ for $R_{0} \leqq R<R_{1}^{(n)}+\rho_{n}$ and further $\lambda_{n}(R) \equiv l_{n}(R)$ for $R_{0}^{(n)} \leqq R \leqq R_{1}^{(n)}$; on $R_{0} \leqq R \leqq R_{0}^{(n)}$ and $R_{1}^{(n)} \leqq R \leqq R_{1}^{(n)}+\rho_{n}, \lambda_{n}(R)$ is to be defined so as to make the boundary smooth at the end points of the corresponding boundary arcs. It is immediately verified that the latter is possible. Let $f_{n}(z)$ be the function mapping $|z|<1$ conformally on $D_{n}$ so that $f_{n}(0)=0, f_{n}^{\prime}(0)>0$.

The function $f_{n}(z)$ may not belong to $Y$ since we may not have $f_{n}^{\prime}(0)=1$. However by our preliminary argument it follows the boundary values of $\Im\left\{z f_{n}^{\prime}(z) / f_{n}(z)\right\}$ are non-negative and extend continuously its values on the interior of the upper semicircle $|z|<1, \Im z>0$. A similar remark applies to the lower semicircle. Thus $f_{n}(z)$ satisfies inequality (2). By the Carathéodory theory of kernels of domains $[1], f_{n}(z)$ and $f_{n}^{\prime}(z)$ taken at any point of $|z|<1$ tend to $f(z)$ and $f^{\prime}(z)$ as $n$ approaches infinity. Thus inequality (2) holds for the function $f(z)$ also. This completes the proof of Theorem 1 .

3. The condition (2) does not of itself imply that a function satisfying it and having the normalization (1) at the origin is univalent, unlike the corresponding condition for starlike functions. It would only imply that the function mapped $|z|<1$ on what might be described as a circularly symmetric Riemann surface, whose description would parallel that given above. Conditions (1) and (2) would provide the complete characterization for such functions. We shall not discuss this possibility further here. To assure univalence it is necessary either to add this condition directly or to add some condition which taken with the inequality (2) implies univalence, for example that the function belong to $T$. We now prove

THEOREM 2. If $f \in Y$, then $f$ maps each circle $|z|<r, 0<r<1$, onto a circularly symmetric domain. 
Indeed, unless $f(z) \equiv z$ in which case the result is obvious, at all points on $|z|=r, \Im z>0$ we have $\Im\left\{z f^{\prime}(z) / f(z)\right\}>0$, thus the image of this open arc by $f$ meets each circle $|w|=R$ at most once and as we describe the arc from $z=r$ to $z=-r$ the circles it meets have decreasing radii. A similar remark applies to the open arc $|z|=r, \Im z<0$. Since the power series expansion of $f(z)$ about $z=0$ has real coefficients this implies that the image domain of $|z|<r$ under $f$ is circularly symmetric.

4. The power series expansion of $z f^{\prime}(z) / f(z)$ about $z=0$ is given, provided $f(z)$ is normalized as in (1), by

$$
z f^{\prime}(z) / f(z)=1+a_{2} z+\left(2 a_{3}-a_{2}^{2}\right) z^{2}+\cdots .
$$

If the function $f \in Y$, provided that $z f^{\prime}(z) / f(z)$ does not reduce to the constant 1 (which is equivalent to $f(z) \equiv z$ ), the inequality (2) implies that the image of the circle $|z|=r$ by this function has degree at most 1 about the point 1 . Thus the coefficient $a_{2}$ cannot vanish. Since $a_{2}$ is real and $f(z)$ attains its maximum modulus for $z$ real and positive we have $a_{2}>0$.

TheOREM 3. If $f \in Y$ either $f(z) \equiv z$ or $a_{2}>0$.

The function $z f^{\prime}(z) / f(z)$ for $f \in Y$ is by the inequality (2) typically real, provided $f(z) \not \equiv z$, apart from lacking the proper normalization at $z=0$. To attain this normalization we form the function $a_{2}^{-1}$ $\cdot\left\{\left(z f^{\prime}(z) / f(z)\right)-1\right\}$ which has expansion about $z=0, z+\left(2 a_{2} / a_{3}-a_{2}\right) z^{2}$ $+\cdots$.

THEOREM 4. $f \in Y$ if and only if either $f(z) \equiv z$ or $f(z)$ and

$$
a_{2}^{-1}\left\{\left(z f^{\prime}(z) / f(z)\right)-1\right\}
$$

are both in $T$.

As a consequence of this result it is possible to give necessary and sufficient conditions on the coefficients of $f$ in order that $f$ be in $Y$. This is done by translating the conditions given by Rogosinski [4, p. 95] for the coefficients of a function in $T$. We shall not give them in extenso here but only point out that it is readily verified that the exact range of values of $a_{2}$ is $0 \leqq a_{2} \leqq 2$ and that Rogosinski's conditions applied to the first nontrivial coefficient of $a_{2}^{-1}\left\{\left(z f^{\prime}(z) / f(z)\right)-1\right\}$ give

$$
\left|2\left(a_{3} / a_{2}\right)-a_{2}\right| \leqq 2
$$

or 


$$
\left|2 a_{3}-a_{2}^{2}\right| \leqq 2 a_{2},
$$

a best possible inequality.

Since functions in $Y$ have real coefficients we have for the latter $\left|a_{n}\right| \leqq n$, and these inequalities are best possible since $z(1-z)^{-2}$ is in $Y$.

Finally it should be observed that certain other of Rogosinski's results can be carried over to give conclusions for functions in $Y$. For example, unless $f(z) \equiv z$, for $f \in Y$ and given $z$ with $|z|<1$ the value $a_{2}^{-1}\left\{\left(z f^{\prime}(z) / f(z)\right)-1\right\}$ lies in that segment of the circle through the points $0, z(1+z)^{-2}, z(1-z)^{-2}$ which corresponds to the arc determined by the two latter points not passing through 0 [4, p. 99]. Also similar theorems can be obtained for functions associated with other forms of symmetrization, for example, Steiner symmetrization.

\section{BIBLIOGRAPHY}

1. C. Carathéodory, Untersuchungen über die konformen Abbildungen von festen und veränderlichen Gebieten, Math. Ann. vol. 72 (1912) pp. 107-144.

2. R. Nevanlinna, Über die konforme Abbildung von Sterngebieten, Översikt av Finska Vetenskaps-Societetens Förhandlingar vol. 63 (1920-1921) Avd. A. No. 6.

3. T. Rad6, Bemerkung ïber die konformen Abbildungen konvexer Gebiete, Math. Ann. vol. 102 (1930) pp. 428-429.

4. W. Rogosinski, Über positive harmonische Entwicklungen und typisch-reelle Potenzreihen, Math. Zeit. vol. 35 (1932) pp. 93-121.

University of Notre Dame 